\title{
Elektronenmikroskopische Befunde zur Morphologie der Cuticula von Blüten gärtnerischer und landwirtschaftlicher Nutzpflanzen
}

\author{
Von Gottfried Bringmann und Renate KühN*** \\ Aus dem Institut für Angewandte Botanik der Technischen Universität Berlin-Charlottenburg \\ und dem Biologischen Laboratorium des Bundesgesundheitsamtes, Institut für Wasser-, \\ Boden- und Lufthygiene, Berlin-Dahlem \\ (Z. Naturforschg. 10 b, 47-58 [1955]; eingegangen am 16. Oktober 1954)
}

\begin{abstract}
Mit Hilfe von Kupferoxydammoniak gelingt es, die Cuticulae der Blütenteile frei von Begleitsubstanzen zu gewinnen. Die isolierten Cuticulae sind im elektronenmikroskopischen Bild von geringem Elektronenkontrast und somit bei $80 \mathrm{kV}$ Strahlspannung in brauchbarer Weise für die elektronenmikroskopische Beobachtung zu durchstrahlen und mit hoher Auflösung abzubilden. Eine unvollständige Ablösung von Fremdsubstanzen ist im Elektronenbild leicht erkennbar und kann somit kontrolliert werden, zumal die Cuticula selbst, weder bei hoher Vergrößerung noch bei Verstärkung des Elektronenkontrastes, eine innere Strukturierung erkennen läßt. In dem homogenen Häutchen konnten auch niemals native Poren sichtbar gemacht werden. Hingegen besitzen die Cuticulae in zahlreichen Fällen eine durch Fältelung bedingte Musterung, deren Feinheiten erst im Elektronenbild sichtbar werden. Das Muster korrespcndiert in den meisten untersuchten Fällen mit dem darunterliegenden Zellgefüge. Es wird versucht, die mannigfache Ausprägung des Fältelungsmusters der Cuticula der untersuchten Blüten in einer Reihe von Typen zu ordnen, wobei die Art der Fältelung, die Ausbildung der Zellgrenzen und die Wölbung bzw. Ausstülpung der Cuticula als kennzeichnend angesehen wurde. Das Fältelungsmuster verschiedener Teile einer Blüte, ja selbst innerhalb des gleichen Blütenteils, kann sehr unterschiedlich sein. Innerhalb der Gattungen ist für einzelne Blütenteile eine Übereinstimmung des Musters feststellbar, ebenso zwischen Kulturformen und Wildformen der gleichen Art. Eine Änderung des Musters tritt bei Polyploidie bei den untersuchten Pflanzen nicht in Erscheinung. Das Fältelungsmuster dürfte erblich festgelegt sein, jedoch durch die jeweilige Größe des Blütenteils modifiziert werden. Trotz der aus zeitlichen Gründen beschränkten Anzahl der untersuchten Pflanzen konnte bereits eine Reihe von Zusammenhängen aufgezeigt werden. Für systematische Analysen der Fältelungsmuster der Cuticulae der Blüten werden weitere umfangreichere Beobachtungen angestrebt.

Mit entsprechenden Präparationsmethoden gelingt es auch, die Exine der Pollen im elektronenmikroskopischen Bild darzustellen (Abb. 14, 15). Über weitere Untersuchungsergebnisse an Pollen berichten wir an anderer Stelle. Hier sei nur bemerkt, daß auf dem Maschengerüst der Exine der Pollen von Hyazinthus warzenförmige Erhebungen mit höherer Elektronenstreuung in variierender Größe sichtbar werden.
\end{abstract}

\section{Methode}

$\mathrm{D}_{\mathrm{r}}^{+\infty}$ as Ziel der vorliegenden Arbeit war es, die morphologische Ausprägung der Cuticula direkt im elektronenmikroskopischen Bild sichtbar zu machen. $\mathrm{Zu}$ diesem Zweck mußte die Cuticula frei von jeglichen Zellulosebestandteilchen gewonnen werden. Zur Isolierung des Häutchens legten wir nicht ganze Organteile in das zellulose-lösende Reagens, sondern versuchten zuvor die Epidermis von dem betreffenden Pflanzenteil abzuziehen oder einen Längsschnitt durch das Organ zu führen. Auf diese Weise war es

* Anschrift: Dozent Dr. rer. nat. G. Bring ma n n, Berlin-Dahlem, Corrensplatz 1. - Dr. agr. Renate K ü hn, Berlin-Dahlem, Hüttenweg 15.

${ }^{* *}$ G. B r in g mann u. R. K ü h n, Atlas der BlütenCuticula (in Vorbereitung). möglich, die Cuticulae einzelner Oberflächenbereiche freizulegen. Nur in wenigen Fällen mußten gesamte Organstückchen mit dem Reagens behandelt werden.

Als Lösungsmittel für Zellulose verwendeten wir Kupferoxydammoniak, ein Reagens, das Cutin nicht angreift (K le in 1, S. 219). Die Auflösung der Zellulose erfolgte bei niedriger Temperatur rascher und vollständiger als bei höherer. Nach einer Einwirkungsdauer von ungefähr 14 Tagen schwammen die isolierten Cuticulae auf der Oberfläche des Lösungsmittels. Zur Lösung noch anhaftender Reste von Kupfersalz wurden die Häutchen mit einer Präpariernadel in 4-proz. Essigsäure übertragen. Häufig fielen erst in der Essigsäure die anhaftenden Gewebsreste von der Cuticula ab. Die schwimmenden Cuticulahäutchen wurden mit Hilfe einer Hakenpinzette auf den Objektträger-Schlitzblenden aus 2-proz.

1 G. K l e i n, Handbuch der Pflanzenanalyse, 3. Band, 2. Teil, 1. Aufl., Wien 1932. 
Essigsäure aufgefangen und die Essigsäure sofort mit Filtrierpapier abgesaugt.

Da uns für die Arbeit am Elektronenmikroskop nur ein halber Arbeitstag in der Woche zur Verfügung stand, war es nicht möglich, Tausende von Präparaten vollzählig elektronenmikroskopisch zu beobachten. Deshalb fand eine lichtmikroskopische Voruntersuchung statt. Hierfür erprobten wir nachstehende Untersuchungsmethoden: Das Häutchen wurde entweder in Polyvinylalkohol oder in Wasser eingebettet und lichtmikroskopisch im Hellfeld bzw. im Phasenkontrast-Mikroskop beobachtet; oder die Häutchen wurden auf Objektträgerblenden aufgefangen und lichtmikroskopisch ohne Einschlußmittel betrachtet. Die letzte Methode lieferte die schnellsten und einwandfreiesten Ergebnisse, wie nachträglich durch elektronenmikroskopische Kontrollbeobachtungen feststellbar war. Wir arbeiteten bei einer 500 -fachen lichtmikroskopischen Vergrößerung. Bei dieser Vergrößerung konnte mit großer Sicherheit unstrukturierte und strukturierte Cuticulae wenigstens in groben Zügen unterschieden werden. Es wurden die strukturierten Cuticulae, die für unsere derzeitigen Untersuchungen von alleinigem Interesse waren, herausgesucht und im Elektronenmikroskop zur Darstellung gebracht. Die Ablösung der Zellulosereste von der Cuticula konnte lichtmikroskopisch nicht erkannt werden. In dem elektronenmikroskopischen Bild waren jedoch nach entsprechender Erfahrung Zellulosereste an der Cuticula ohne weiteres erkennbar. War die Zellulose noch nicht vollständig von dem Präparat abgelöst. so übertrugen wir die Parallelpräparate noch einmal aus der Essigsäure in Kupferoxydammoniak. In diesem Lösungsmittel verblieben die Präparate dann nochmals 14 Tage.

Als Reagenzien für die Isolierung der Cuticula werden in der Literatur 85-proz. Phosphorsäure und Eau de Javelle angeführt. Um diese Lösungsmittel auf ihre Eignung für unsere Zwecke zu erproben, übertrugen wir Präparate, die bereits mit Kupferoxydammoniak weitgehend, jedoch nicht vollständig, von der anhaftenden Zellulose befreit worden waren, aus Essigsäure über Wasser in die angeführten Lösungsmittel. Jedoch das elektronenmikroskopische Bild zeigte, daß die Präparate durch die Behandlung mit diesen Lösungsmitteln nicht weitergehend von der anhaftenden Zellulose befreit wurden. Außerdem beobachteten wir Stoffablagerungen auf den Präparaten, die wahrscheinlich aus den Lösungsmitteln stammten. Nebenher konnte bei einer Reihe von Präparaten, deren ungestörte Ausprägung uns bereits bekannt waren, strukturelle Störungen beobachtet werden. Auf Grund dieser Versuchsergebnisse verwendeten wir in der Folge nur noch Kupferoxydammoniak als Lösungsmittel.

Für die elektronenmikroskopischen Untersuchungen stand ein Siemens-Übermikroskop, Typ $100 \mathrm{~b}$, für 60 bis $100 \mathrm{kV}$-Strahlspannung zur Verfügung. Zur Durchstrahlung unserer Cuticulapräparate war die Strahlspannung von $80 \mathrm{kV}$ vollkommen ausreichend.

Das Prinzip der elektronenmikroskopischen Darstellung ist die Abbildung aller Strukturen, die mit einer variierenden Massendicke parallel gehen. Bei der Durchstrahlung der Cuticula-Präparate im Elektronenmikroskop erfolgt demnach die reine Darstellung der Massendicke des Cutins. Eine hohe Elektronenstreuung zeigt einen Bereich dicker bzw. mehrfach überlagerter Cutin-Schichten an. Auf den elektronenmikroskopischen Aufnahmen kann man mithin aus den Abstufungen der Schwärzung die unterschiedlichen Dickenverhältnisse des Cutins ersehen, vorausgesetzt, daß alle Begleitstoffe entfernt worden sind. Der hohe Vorteil der 100-fach höheren Auflösung der elektronenmikroskopischen Darstellungsmethode wird besonders deutlich an Vergleichsaufnahmen von Cuticula-Präparaten im Lichtmikroskop bei 500-facher Vergrößerung und anschließender Aufnahme der gleichen Stellen im Elektronenmikroskop (Abb. 12, 13).

\section{A rbeits gang}

Da Lee und Priestley ${ }^{2}$ (S. 531) fanden, daß der ganze Embryo, d. h. Kotyledonen, Plumula und auch die Radicula von einer Cuticula überzogen sind, begannen wir unsere Untersuchungen an den Cuticulae von Embryonen. Für diese Untersuchungen wurden zunächst aus ruhenden Samen der Buschbohne, Markerbse, Tomate und des Weißkohls die Kotyledonen und die Radicula herauspräpariert. Außerdem haben wir von den beiden erstgenannten Gemüsearten die Primärblätter, aus dem Samenkorn der Buschbohne, Tomate und Weißkohl noch das Hypokotyl isoliert. Doch es gelangt nicht, von einem dieser genannten Pflanzenteile ein Cuticula-Präparat zu erhalten, da offenbar in Kupferoxydammoniak ein Zerfall eintrat.

Die ersten elektronenmikroskopischen Beobachtungen machten wir daher an den Cuticulae der Sprosse von Jungpflanzen verschiedener Gemüsearten (Gurke, Salat, Kohlrabi, Tomate) in einem Entwicklungszustand, in dem die Kotyledonen vollkommen entfaltet und 1-2 Laubblatt-Paare in der Entwicklung begriffen waren.

Wir erzielten folgende Ergebnisse: Abgesehen von Spaltöffnungen erscheinen die Cuticulae der Ober- und Unterseite von Kotyledonen und Laubblättern sowie die Cuticula der Blattstiele im Elektronenbild nicht strukturiert. Auch das Hypokotyl von Gurke, Salat und Tomate sowie das Epikotyl letztgenannter Gemüseart enthielten keine elektronenmikroskopisch sichtbaren Strukturen.

Die Ober- und Unterseite des ersten Laubblattes und die Innen- und Außenseite der Koleoptile eines Weizenkeimlings fanden wir ebenfalls nicht strukturiert, weder bei hoher Vergrößerung (20000-fach) noch bei Erhöhung des Elektronenkontrastes durch Herabsetzung der kVZahl. Auch Poren waren nicht zu sehen.

Erst bei den Untersuchungen der Cuticula der Blütenteile beobachteten wir Strukturen. Doch bevor von den

2 B. Lee u. J. H. Priestley, Ann. Botany 38, $525[1934]$. 


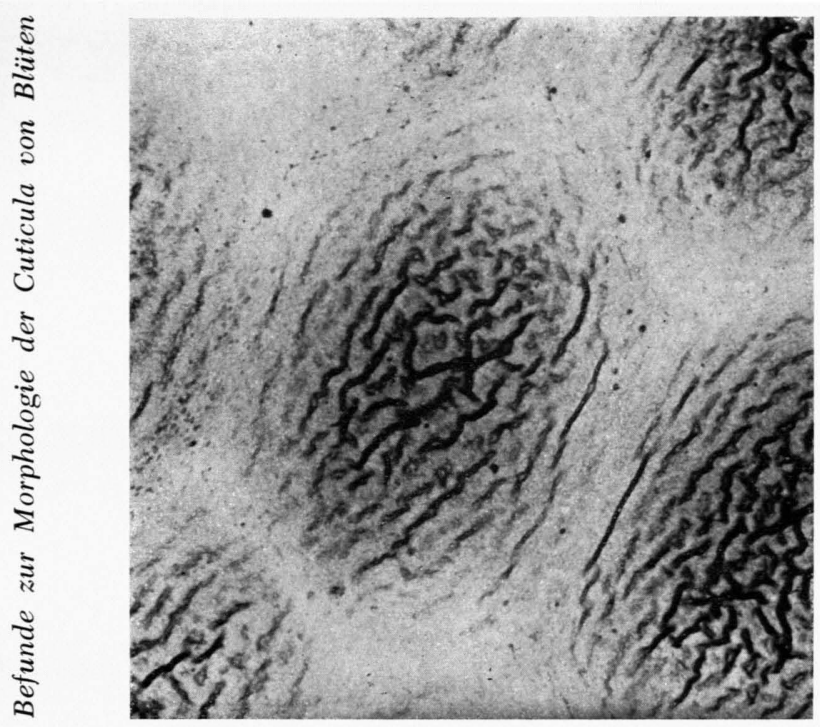

Abb. 3. Cuticula der Oberseite eines inneren Blütenblattes von Azalea spec. Elektronenbild 1500 : 1, $80 \mathrm{kV}$.

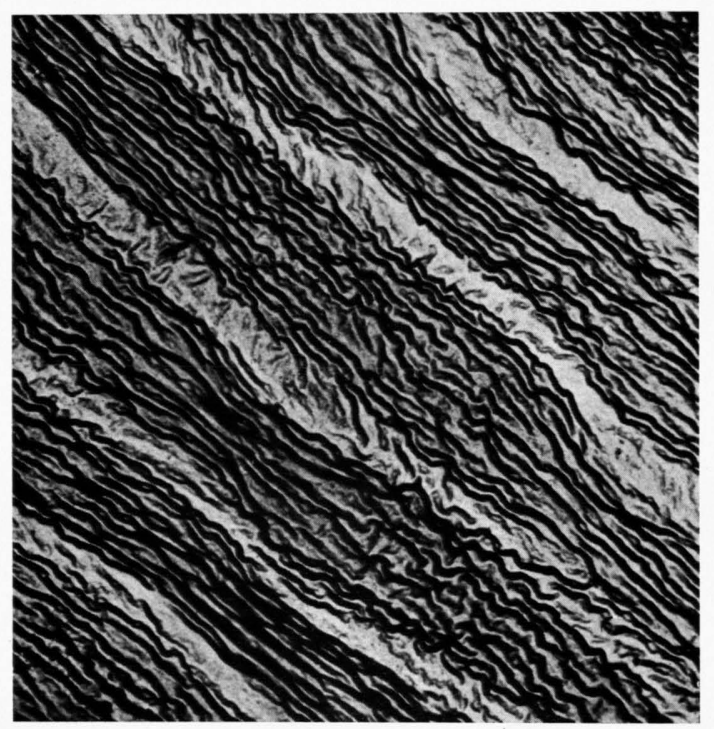

Abb. 5. Cuticula des Griffels von Rubus idaeus. Elektronenbild $1500: 1,80 \mathrm{kV}$

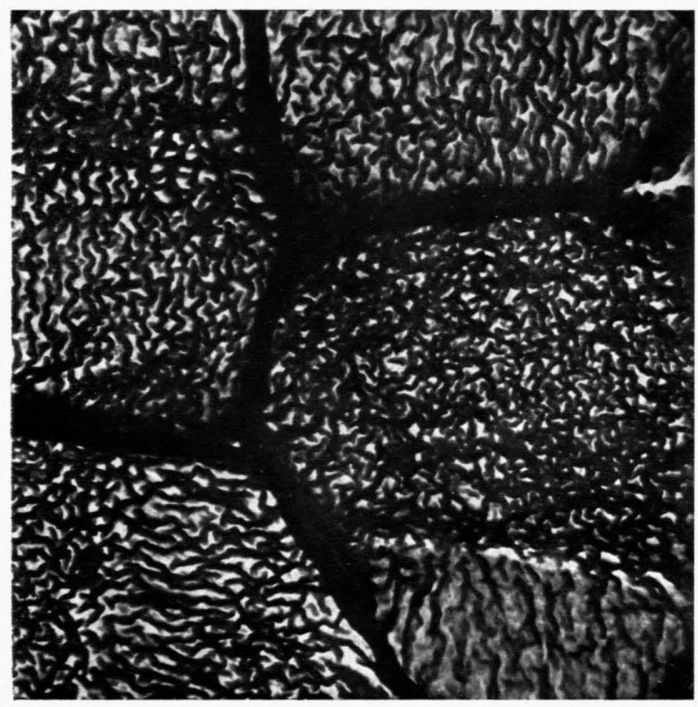

Abb. 7. Cuticula der Außenseite der Staubgefäße von Cyclamen persicum. Elektronenbild 1500: 1, $80 \mathrm{kV}$.

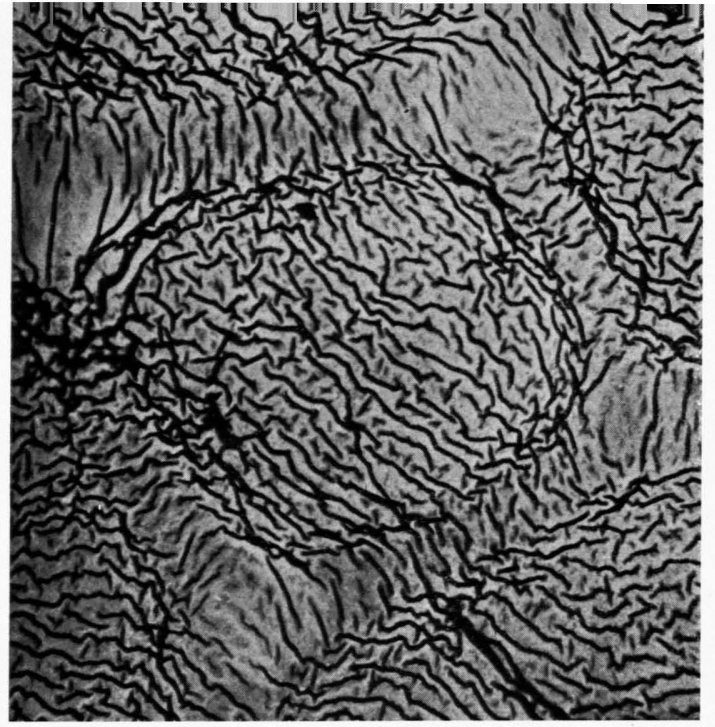

Abb. 4. Cuticula der Innenseite des Flügels von Wistaria sinensis. Elektronenbild $1500: 1,80 \mathrm{kV}$.

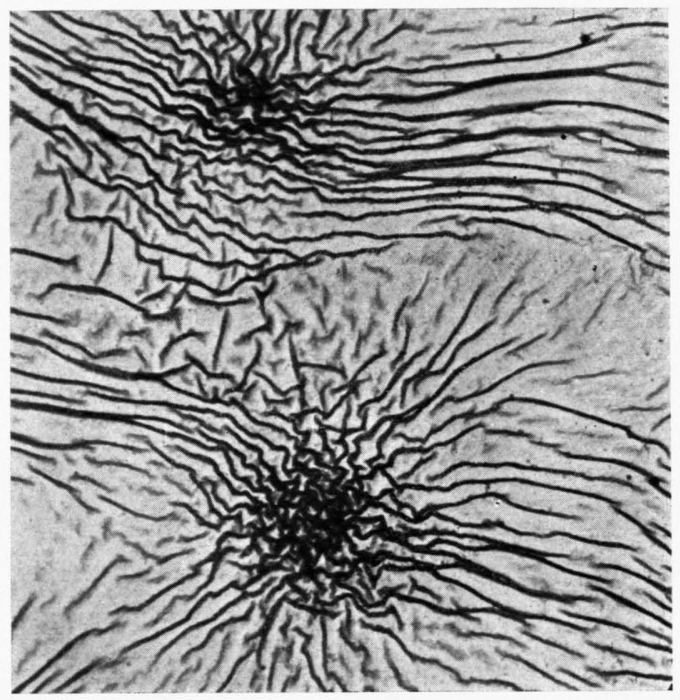

Abb. 6. Cuticula der Außenseite des Flügels von Wistaria sinensis. Elektronenbild $1500: 1,80 \mathrm{kV}$.

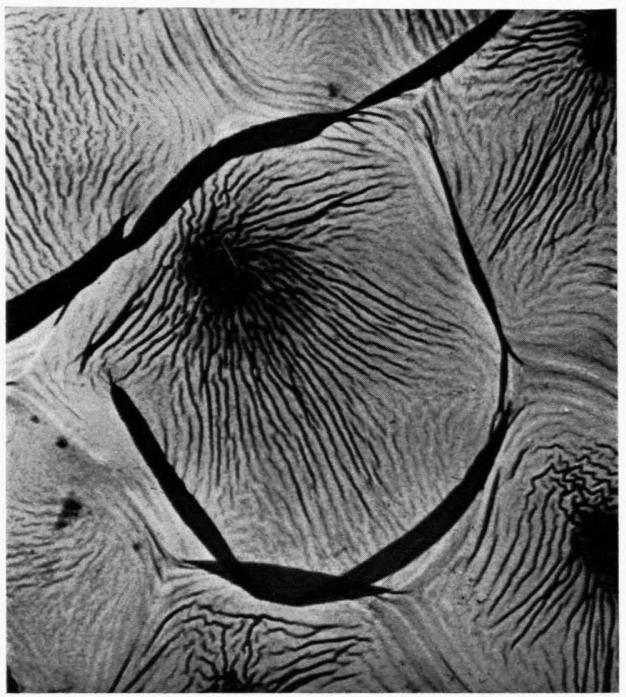

Abb. 8. Cuticula der Innenseite des Flügels von Lupinus luteus. Elektronenbild $1500: 1,80 \mathrm{kV}$. 
eigenen Untersuchungen berichtet wird, soll auf die in der Literatur vorliegenden Ergebnisse eingegangen werden.

$$
\text { Die Cuticula der Blüten }
$$

1884 schreibt $\mathrm{Hill}_{\text {er }}{ }^{3}$ (S. $419 \mathrm{ff}$.) über Untersuchungen an der Epidermis der Blütenblätter u. a.: „Am häufigsten ist die gestreift-gerippte Cuticula, welche längs und radiär verlaufende rippenartige Erhebungen von verschiedener Größe und Form besitzt. Seltener findet man, daß sie knötchenartige Verdickungen zeigt.“ „Die Rippen selbst können entweder gerade oder gebrochene Linien darstellen. Sie ziehen sich manchmal über mehrere Zellen ohne Unterbrechung hinweg und verdicken sich dann wohl auch an den Kreuzungspunkten mit den EpidermisZellwänden“, „beschränken sich aber auch oft auf eine einzige Zelle. Verlaufen sie radiär,“ „so beginnen sie an einem Punkte, der meist in der Mitte der Zelle, selten exzentrisch gelegen ist. Dieser Fall findet wohl bei allen papillösen Zellen statt, wo dann die Rippen an der Papille herablaufen.“

Volkens (1884, S. 14) (bei Lin s ba u e r ${ }^{4}$, S. 95) gibt für manche einheimische Orchideen an, daß die Cuticulafalten gerade über den Radialwänden stärker ausgebildet oder sogar auf diese Stellen beschränkt seien, während Vesque (1882) (bei Lins baue r ${ }^{4}$, S. 95) anführt, daß die Faltenbildung bevorzugt auf den linsenartig nach außen vorgewölbten Epidermiszellen stattfindet. Solereder ${ }^{5}$ (S. 579) spricht bei Beschreibung der Cuticulastruktur von Laubblattepidermen verschiedener Pflanzengattungen von „Körnung, Streifung“ und „Leisten- oder Kammbildung“. Eine „körnige geschichtete“ Cuticula beschreibt Hering ${ }^{6}$ (S. 8) an der Infloreszenzachse von Hygrochilus parishii ( $\mathrm{Pf}$ it $\mathrm{z})$.

L in s b a u e ${ }^{4}$ (S. 94) teilt die Strukturen der Cuticula in zwei Typen ein:

1. die Faltenbildungen (Streifen, Wellen),

2. die Protuberanzen (Höcker, Warzen).

Auf die Faltenbildungen der Cuticula wurde bereits in den zitierten Literaturstellen näher eingegangen.

Als Protuberanzen erklärt $\mathrm{L}$ in s b a u e $r$ papillöse Bilàungen, die lediglich aus Cutin bestehen oder Membranzapfen, die nur von einer Cuticula umkleidet sind. Erstere fand Hering ${ }^{6}$ (S. 73) am Stamm von Vanda teres. Auch an Gramineen oder Cyperaceen sind sie vorhanden.

Doch all diesen Ausführungen sind, entsprechend der damaligen Darstellungsweise, Zeichnungen lediglich von der Oberfläche und von Querschnitten durch die Epidermis beigefügt, die aber in keiner Weise die Feinheiten der Cuticulastruktur, wie sie oben beschrieben werden. wiedergeben.

Erst F e ld h of e ${ }^{7}$ (S. 499, 512) arbeitete mit isolierten Cuticula-Präparaten. Er isolierte diese von Nektarien verschiedener Pflanzen, um an ihnen nach Durchtrittsstellen für den Nektar zu suchen.

3 G. H. H ill e r, Jb. wiss. Bot. 15, 411 [1884].

$4 \mathrm{~K}$. L i n s b a u e r, Handbuch der Pflanzenanatomie, Band IV, Die Epidermis. 1. Aufl., Berlin 1930.

$5 \mathrm{H}$. S ol ed erer, Systematische Anatomie der Dicotyledonen. 1. Aufl., Stuttgart 1899.
Volz ${ }^{8}$ (Seite 261-263) untersuchte als erste elektronenmikroskopisch isolierte Cuticulahäutchen von Blütenblatt, von Laubblättern und Nektarien. Doch kommt Volz zu dem Schluß, daß die Cuticulae der meisten von ihr untersuchten Laubblätter zu dick für Beobachtungen im Elektronenmikroskop seien oder nur „schwer deutbare Strukturen“ hätten.

Entgegen diesen Feststellungen fanden wir bei der Durchsicht mehrerer tausend Präparate von Cuticulae der Blüten-Region nicht ein einziges Präparat mit undurchstrahlbaren Cuticulahäutchen.

Bedingt durch die Jahreszeit begannen wir unsere Arbeiten an Blüten von Zierpflanzen; im Laufe der Sommermonate bearbeiteten wir Blüten landwirtschaftlicher und gärtnerischer Nutzpflanzen. Bei der Auswahl des Materials griffen wir aus lagebedingten Gründen auf solche Nutzpflanzen zurück, die in den Instituten der Fakultät für Landbau der Technischen Universität Berlin-Charlottenburg zur Verfügung standen.

Von den Blüten wurden im allgemeinen folgende Teile untersucht:

I. Perianth: 1. Sepalen (Oberseite und Unterseite), 2. Petalen (Oberseite und Unterseite) bzw. Tepalen (Oberseite und Unterseite).

II. St a m en : 1. Filament, 2. Antheren (wenn präparativ möglich, Theken vom Konnektiv getrennt).

III. Fruchtknoten: 1. Ovarium, 2. Stylus, 3. Stigma.

Im einzelnen wurde noch unterschieden auf dem Blütenblatt zwischen dem Saftmal (z. B. bei Primel, Puffbohne) und dem übrigen Blütenblatt.

Bei den Sympetalen beobachteten wir die Blütenröhre getrennt von den Blütenblatt-Spreiten (z. B. bei Forsythia).

In die Untersuchungen wurden auch spezielle Blütenblatt-Bildungen, wie der Sporn von Viola, die Spatha von Zantedeschia oder das Blütenhüllblatt von Helleborus, ferner das Gynostemium der Orchidee, der Blütenboden, insbesondere der Rosaceen, und florale Nektarien miteinbegriffen.

In Tab. 1-3 sind die von 67 Pflanzen gewonnenen Ergebnisse unter dem Gesichtspunkt zusammengestellt, ob die einzelnen Blütenteile Cuticula-Strukturen oder unstruktierte Cuticula aufweisen. Aus dem Vergleich derselben Blütenteile verschiedener Arten ergibt sich, daß die Cuticula jedes Blütenteils in einem Fall strukturiert, im anderen Falle vollkom-

6 J. H e rin g, Bot. Zbl. 83, 73 [1900].

7 E. Feldhofen, Beih. bot. Zbl., Abt. A 50, 459 [1933].

8 G. V o l z, Mikroskopie 7, 251 [1952]. 
men strukturlos sein kann. Aus der Tabelle ist weiterhin zu entnehmen, daß innerhalb einer Blüte das eine Organ eine Cuticula-Struktur besitzen kann, während ein anderer Blütenteil eine unstrukturierte Cuticula aufweist. Selbst hinsichtlich Kron- bzw. Kelchblatt-Ober- und Unterseite ergeben sich charakteristische Unterschiede.

Als Beispiele möchten wir anführen: Es sind bei Linum, bei Papaver, Cucumis, Juglans regia, Eranthis, Anemone hepatica die Cuticula des Perianth, der Stamina und des Fruchtknotens vollkommen unstrukturiert.

Im Gegensatz dazu sind bei Hydrangea die Cuticulae sämtlicher Blütenteile strukturiert.

Wistaria und Freesia besitzen demgegenüber struk-

a)

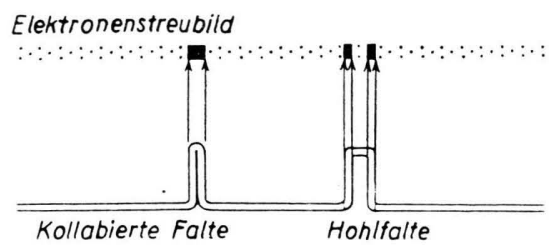

b)

Elektronenstreubild

Abb. 1. Schematische Darstellung der Entstehung des Elektronenbildes von Faltentypen der Cuticula.

turierte Cuticulae nur an den Blütenblättern; Tomate und Kartoffel an den Antheren und am Griffel und Secale, Triticum, Triticale, Avena und Hippeastrum nur an den Antheren.

Wir kamen zunächst auf Grund unserer Untersuchungen zu dem Schluß, daß sich (mindestens bei den von uns untersuchten Blüten) die in der Musterung der Cuticula abzeichnenden Zellgrenzen mit den darunterliegenden Wänden der Epidermiszellen übereinstimmen. Auf den elektronenmikroskopischen Bildern von Cuticulae, deren Zellulosebegleitsubstanz nicht völlig abgelöst ist, sind die darunterliegenden Zellgrenzen durch einen hohen Elektronenkontrast gegenüber dem Lumen ausgezeichnet. So erkennbare Zellgrenzen deckten sich in fast allen Fällen mit einer zellulär ausgeprägten Strukturierung der Cuticula (Abb. $3^{*}$ und 4).

* Abb. 3-15 s. Tafel S. 48 a u. b.
Bei der Auswertung von Elektronenbildern der Cuticula ist zu beachten, daß das ursprünglich dreidimensionale Relief der Cuticula der turgeszenten Zellen auf Grund der Wirkung der Präparation und der Eigenart des Darstellungsverfahrens praktisch in eine Ebene projiziert wird. Vertikal stehende Wände feiner Cuticulafältchen erscheinen auf Grund ihrer höheren Elektronenstreuung dunkel; alle horizontal liegenden Teile der Falten und der Grundcuticula hingegen auf Grund der geringeren Elektronenstreuung hell (Abb.1a). Mehr oder minder vertikal gerichtete Cuticulateile turgeszenter Zellen mit linsen-

a)

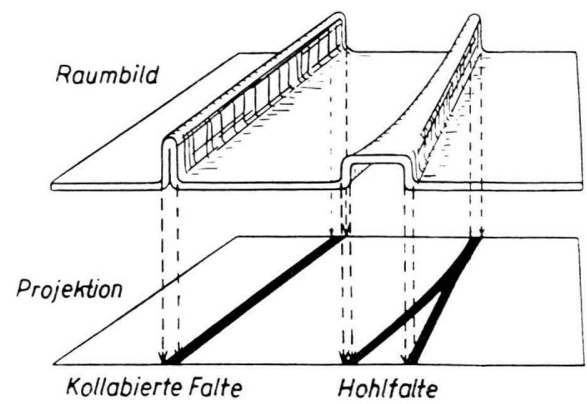

b)

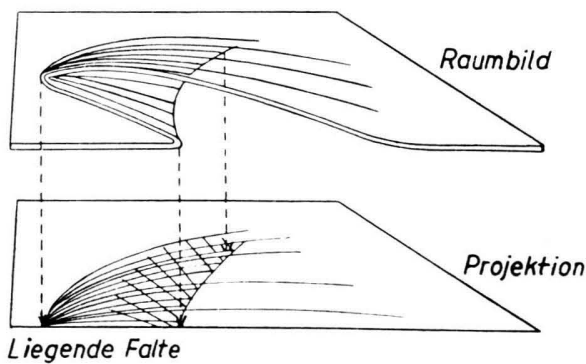

Abb. 2. Schematische Darstellung des Raumbildes und des Projektionsbildes von Faltentypen der Cuticula.

förmiger Oberfläche bzw. die Cuticula von Papillen treten bei frei präparierten Cuticulae als Úberlagerungsfalten in Erscheinung (Abb. 2b), die infolge ihrer Mehrschichtigkeit einen höheren Elektronenkontrast geben als die einfache Cuticula (Abb.1b). Wir kommen zu der Auffassung, daß die Strukturie-

Erklärungen der Abkürzungen und Zeichen in den Tab.:

$$
\begin{aligned}
\mathrm{US} & =\text { Unterseite, } \\
\mathrm{OS} & =\text { Oberseite } \\
\mathrm{AS} & =\text { Außenseite, } \\
\mathrm{JS} & =\text { Innenseite, } \\
\mathrm{P} & =\text { Papillen, } \\
\mathrm{T} & =\text { Trichome }, \\
+ & =\text { gefältelte Cuticula }, \\
- & =\text { nicht gefältelte Cuticula, } \\
\text { Nag. } & =\text { Nagel, } \\
\text { get. B. } & =\text { getigerte Basis. }
\end{aligned}
$$




\begin{tabular}{|c|c|c|c|c|c|c|c|c|c|c|c|c|c|c|c|c|c|c|c|c|c|c|c|c|c|}
\hline \multirow[t]{2}{*}{ Pflanzenname } & \multirow{2}{*}{\multicolumn{2}{|c|}{ 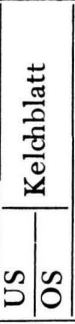 }} & \multicolumn{4}{|c|}{ Blütenblatt } & \multicolumn{2}{|c|}{ 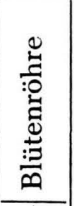 } & \multicolumn{3}{|c|}{ 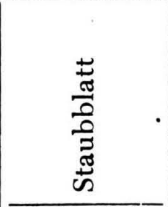 } & \multicolumn{3}{|c|}{ 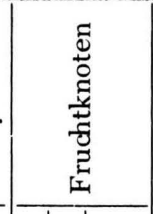 } & \multirow{2}{*}{$\begin{array}{l} \\
\dot{z} \\
\text { ż } \\
- \\
0 \\
0\end{array}$} & \multirow{2}{*}{ 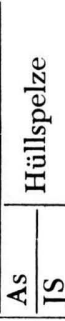 } & \multirow{2}{*}{\multicolumn{2}{|c|}{ 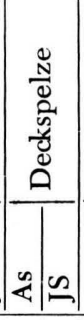 }} & \multirow{2}{*}{$\begin{array}{l}\frac{N}{0} \\
\frac{0}{0} \\
\frac{0}{2} \\
1 \\
\end{array}$} & \multirow{2}{*}{$=$} & \multirow{2}{*}{ | } & \multirow{2}{*}{ |. } & \multirow{2}{*}{ 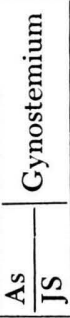 } & \multirow{2}{*}{\multicolumn{2}{|c|}{$\frac{\underset{\pi}{\pi}}{\text { की }}$}} \\
\hline & & & $\begin{array}{c}\text { US } \\
(\mathrm{AS})\end{array}$ & $\begin{array}{c}\text { OS } \\
\text { (JS) }\end{array}$ & $\begin{array}{c}\text { US } \\
\text { (AS) }\end{array}$ & $\begin{array}{c}\text { OS } \\
\text { (JS) }\end{array}$ & $\mid 20$ & $\stackrel{\infty}{\infty}$ & 宦 & $\frac{\vec{v}}{\mathrm{E}}$ & $\begin{array}{l}\dot{0} \\
0 \\
\vdots \\
\mid\end{array}$ & $\begin{array}{l}3 \\
0\end{array}$ & ن & ż & & & & & & & & & & & \\
\hline Tulipa spec. . . . . . . . . . . & & & + & + & & & & & + & + & + & - & & $-\mathrm{P}$ & & & & & & & & & & & \\
\hline Scilla sibirica. . . . . . . . . . . . & & & & + & & & & & + & + & & - & + & & & & & & & & & & & & \\
\hline Muscari botryoides . . . . . . . . . & & & - & $-\quad+$ & & & & & & + & & +- & + & & & & & & & & & & & & \\
\hline Asparagus officinalis. . . . . . . . . & & & & - & & & - & + & + & + & & - & & & & & & & & & & & & & \\
\hline Convallaria majalis . . . . . . . . . . . & & & & + & & & & & + & + & & - & - & - & & & & & & & & & & & \\
\hline Hyazinthus orientalis . . . . . . . . & 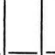 & & & + & & & - & + & + & & & $-1-$ & + & + & & & & & & & & & & & \\
\hline Galanthus nivalis . . . . . . . . . . & & & $+\mathrm{P}$ & + & + & + & & & & & & $-1-$ & + & & + & & & & & & & & & & \\
\hline Narcissus (Trompetennarzisse) . . . . & & 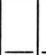 & + & $+\mathrm{P}$ & + & + & & & + & + & & - & $\cdots$ & $+\mathrm{P}$ & & & & & & & & & & & \\
\hline Hippeastrum (Hybride) . . . . . . . & & & $\cdots$ & - & & & & & - & + & + & - & - & $-\mathrm{P}$ & & & & & & & & & & & \\
\hline Crocus spec. . . . . . . . . . . . . & & 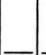 & & - & & & & & $-\mathrm{P}$ & $\cdots$ & - & - & - & - & & & & & & & & & & & \\
\hline 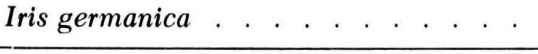 & & & (get. B. - -) & (get. B. - ) & (get. B. - ) & $($ get. B. - ) & - & + & + & + & 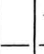 & $-1-$ & + & + & & & & & & & & & & & \\
\hline Freesia spec. . . . . . . . . . . . . . & - & - & + & + & & & - & - & - & - & 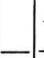 & - & & & & & & & & & & & & & \\
\hline Avena sativa („Flemmings Gold“) . . . & & & & & & & & & & + & - & - & & & & - & - & - & - & - & - & & & & \\
\hline Secale cereale (Stamm 54,106) & & & & & & & & & - & + & & - & & & & - & - & - & - & - & - & - & & & \\
\hline Triticum sativum („Trumball“) . . . . & & & & & & & & & & + & & - & & & & - & - & 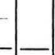 & - & - & - & - & & & \\
\hline Triticale. & & & & & & & & & - & + & - & - & & & & - & - & - & - & - & - & - & & & \\
\hline Hordeum sativum („Heines Haiser II“) & & & & & & & & & & & & - & & & & - & - & - & - & - & - & . & & & \\
\hline Cymbidium (Hybride). . . . . . . . & & & - & -- & $\cdots$ & - & & & & & & - & & & & & & & & & & & - & & \\
\hline Zantedeschia aethiopica . . . . . . . & & & & & & & & & - & $-\mathrm{P}$ & & & +1 & & & & & & & & & & & & $-1-$ \\
\hline
\end{tabular}

Tab. 1. Verteilung gefältelter und nicht gefältelter Cuticula auf den Blütenteilen einiger Monocotylen. 


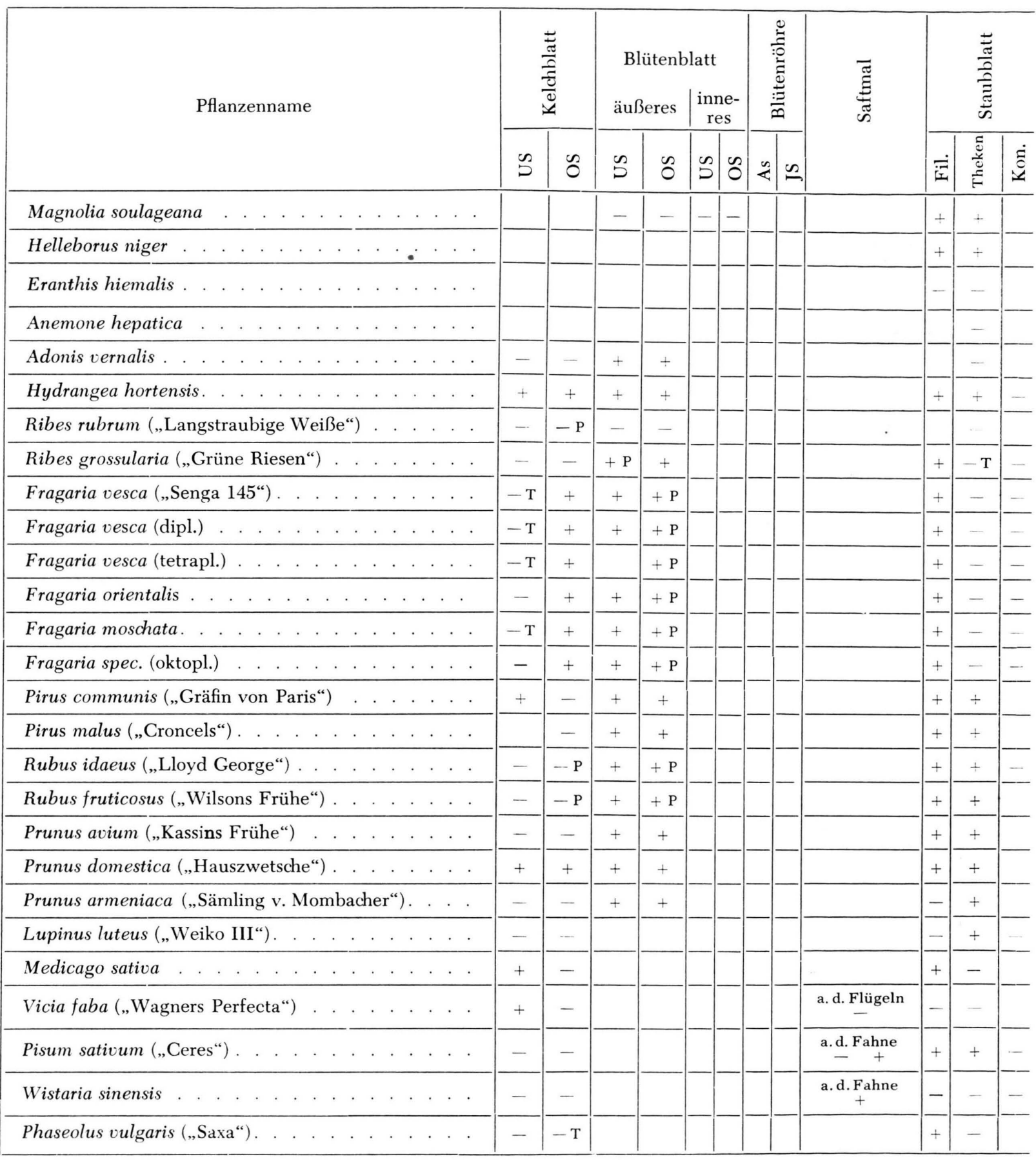

Tab. 2. Verteilung gefältelter und nicht gefältelter

rung der Cuticula durch Fältelung bewirkt wird und nicht durch Rippenbildung. Als Beweis für unsere Anschauung möchten wir anführen:

Die durch höhere Elektronenstreuung charakterisierten Zeichnungen der Cuticula streichen in den meisten Fällen nicht flächenhaft homogen aus, sondern teilen sich in zwei lineare Begrenzungsstrukturen (Abb. 3). Ein solches Ausstreichen ist nur erklärlich, wenn es sich bei den Feinstrukturen um Falten handelt deren Doppelwandigkeit nur dadurch nicht 


\begin{tabular}{|c|c|c|c|c|c|c|c|c|c|c|c|c|c|c|c|c|}
\hline & 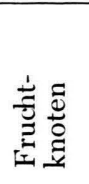 & & & 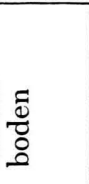 & 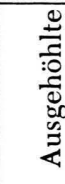 & 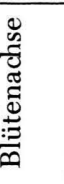 & & 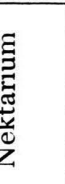 & : & 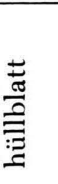 & & hne & & igel & Schi & fchen \\
\hline$\stackrel{\dot{E}}{0}$ & 苛 & $\begin{array}{l}0 \\
\frac{0}{\pi} \\
z \\
Z\end{array}$ & $\sum^{\infty}$ & $\stackrel{\Omega}{=}$ & '先 & $\stackrel{\Omega}{\sim}$ & \& & $\approx \tilde{\theta}$ & $s$ & o & 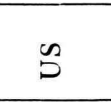 & ? & 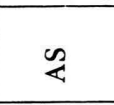 & n & $\frac{n}{4}$ & $\stackrel{n}{.}$ \\
\hline- & - & & & + & & & & & & & & & & & & \\
\hline- & + & & & & & & + & + & + & - & & & & & & \\
\hline$(\overline{\text { Stiel }}-)$ & - & & & & & & $\overline{\overline{(S t i}}$ & $\overline{-1}$ & - & - & & & & & & \\
\hline- & - & & & & & & & & - & $-P$ & & & & & & \\
\hline$-\mathrm{P}$ & - & $-P$ & & & & & & & & & & & & & & \\
\hline+ & + & + & & & & & & & & & & & & & & \\
\hline- & $-P$ & $-P$ & & $-\mathrm{P}$ & & & & & & & & & & & & \\
\hline$-\mathrm{T}$ & $+\quad$ & 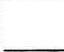 & & $-\mathrm{P}$ & $-\mathrm{T}$ & $-P$ & & & & & & & & & & \\
\hline- & + & + & - & $+\quad-$ & & & & & & & & & & & & \\
\hline- & + & & $-\mathrm{T}$ & & & & & & & & & & & & & \\
\hline- & + & & $-\mathrm{T}$ & & & & & & & & & & & & & \\
\hline - & + & & - & & & & & & & & & & & & & \\
\hline- & + & & $-\mathrm{T}$ & & & & & & & & & & & & & \\
\hline - & + & & $-\mathrm{T}$ & & & & & & & & & & & & & \\
\hline- & - & + & & & & & & - & & & & & & & & \\
\hline- & + & + & & & & & & & & & & & & & & \\
\hline- & + & & & + & & & & & & & & & & & & \\
\hline- & + & & & - & & & & + & & & & & & & & \\
\hline- & + & - & & - & & & & + & & & & & & & & \\
\hline- & + & - & & $+\quad-$ & & & & + & & & & & & & & \\
\hline$-\mathrm{T}$ & + & - & & - & & & & & & & & & & & & \\
\hline- & + & & & & & & & & & & $+\mathrm{P}$ & $+\mathrm{P}$ & $+\mathrm{P}$ & + & - & - \\
\hline- & - & & & & & & & & & & + & $+\mathrm{P}$ & + & $+\mathrm{P}$ & + & + \\
\hline$-P$ & + & $+\mathrm{T}$ & & & & & & & & & $\stackrel{+}{(\text { Nag. }-)}$ & $\begin{array}{c}+ \\
(\text { Nag. }\end{array}$ & $\begin{array}{c}+\mathrm{P} \\
(\mathrm{Nag} .-)\end{array}$ & $\begin{array}{c}+ \\
(\text { Nag. }\end{array}$ & $(\mathrm{Nag} .-)$ & $(\mathrm{Nag} .-)$ \\
\hline- & - & & & & & & & & & & $(\stackrel{+}{+}-)$ & + & $(\stackrel{+}{+}++)$ & $\stackrel{+}{\text { Nag. }} \cdot \overline{+})$ & + & + \\
\hline- & & & & & & & & & & & + & + & + & + & + & + \\
\hline$+P$ & + & & & & & & & & & & + & + & + & - & - & - \\
\hline
\end{tabular}

Cuticula auf den Blütenteilen einiger Dicotylen.

in Erscheinung tritt, daß die kollabierten Wände der Falte als einheitliches Objekt elektronenstreuend in Erscheinung treten. Erst beim Ausstreichen der relativ hohen Falten wird der Elektronenkontrast so vermindert, daß die beiden Vertikalwände der Falte als
Einzellinien in der Projektion in Erscheinung treten können. Bei relativ breiten Falten tritt keine Kollabierung der Faltenwände ein. Auch kann angenommen werden, daß die Falten-Innenräume auf Grund ihres weiteren Lumens durch die Präparation 


\begin{tabular}{|c|c|c|c|c|c|c|c|c|c|c|c|c|c|c|c|c|c|c|c|c|c|c|}
\hline \multirow[t]{2}{*}{ Pflanzenname } & \multicolumn{2}{|c|}{$\begin{array}{l}\frac{ \pm}{\pi} \\
\frac{\pi}{0} \\
\frac{0}{0} \\
01\end{array}$} & \multicolumn{4}{|c|}{ 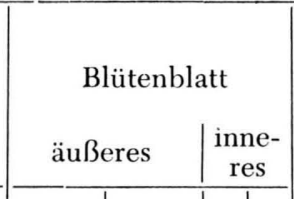 } & \multicolumn{2}{|c|}{ 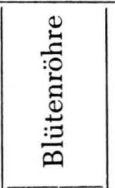 } & \multirow[t]{2}{*}{ 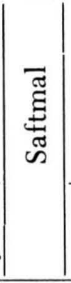 } & \multicolumn{3}{|c|}{ 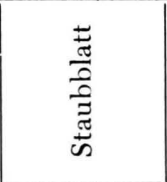 } & \multicolumn{3}{|c|}{ 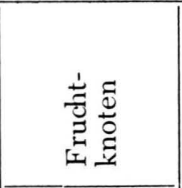 } & \multirow{2}{*}{$\left|\begin{array}{c}\frac{\dot{z}}{0} \\
z \\
\tilde{z} \\
0\end{array}\right|$} & \multicolumn{2}{|c|}{ 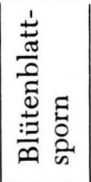 } & 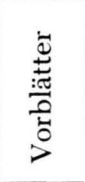 & \multicolumn{3}{|c|}{ 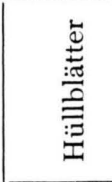 } \\
\hline & $\mathscr{D}$ & $\tilde{0}$ & $\begin{array}{c}\text { US } \\
\text { (AS) }\end{array}$ & \begin{tabular}{l|} 
OS \\
(JS)
\end{tabular} & $\mid$ & $\mathscr{0}$ & $\frac{n}{4}$ & 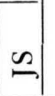 & & $\dot{\overrightarrow{|c|}}$ & 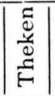 & $\left|\begin{array}{c}\dot{\dot{b}} \\
0 \\
1\end{array}\right|$ & $\begin{array}{l}\dot{\pi} \\
\dot{0}\end{array}$ & 苞 & $\frac{0}{0}$ & & 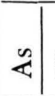 & $\cong$ & $\pi^{2}$ & $2 \frac{2}{4}$ & $\frac{n}{4}$ & $\stackrel{n}{n}$ \\
\hline Papaver somniferum . . . . . . . . . . . . . . . . . & - & - & - & - & & & & & & - & - & & - & & - & & & & & & & \\
\hline Brassica rapa („Lemkes W. Rübsen“) . . . . . . . . & - & - & $\stackrel{+}{+}$ & $\begin{array}{c}+\mathrm{P} \\
(\text { Nag. }-) \\
\end{array}$ & & & & & & -+ & + & - & - & + & - & & & & & & & \\
\hline Brassica napus (Stamm V) . . . . . . . . . . . . . . . & - & - & $\begin{array}{c}+ \\
(\mathrm{Nag} \cdot-\end{array}$ & $\begin{array}{c}+\mathrm{P} \\
(\text { Nag. }-)\end{array}$ & & & & & & + & + & & - & + & - & & & & & & & \\
\hline Viola tricolor („Schweizer Riesen“) . . . . . . . . . . & $\cdots$ & - & $\begin{array}{c}+\mathrm{P} \\
(\mathrm{Nag} \cdot-) \\
\end{array}$ & $\begin{array}{c}++ \\
(\text { Nag. }-) \\
\end{array}$ & & & & & $+\mathrm{P}$ & & + & & & & $+\mathrm{P}$ & + & - & + & & & & \\
\hline Begonia spec. (weibliche Blüte) . . . . . . . . . . . & & & + & + & & & & & & & & & - & + & + & & & & & & & \\
\hline Abutilon spec. . . . . . . . . . . . . . . . . . & - & - & - & - & & & & & & $\cdots$ & & & $-\mathrm{T}$ & - & & $-\mathrm{T}$ & & & & & & \\
\hline Linum usitatissimum (,Vanita“) . . . . . . . . . & - & - & - & - & & & & & & - & - & & - & - & - & & & & & & & \\
\hline Juglans regia . . . . . . . . . . . . . . . . . . . . . . . & & & - & - & & & & & & & - & - & - & & & & & & - & 一 & & \\
\hline Azalea spec. . . . . . . . . . . . . . . . . . & & + & + & + & + & + & + & + & + & & -1. & & $-\mathrm{P}$ & + & - & & & & & & & \\
\hline Primula sinensis . . . . . . . . . . . . . . . . . . . . . & $\mathrm{T}$ & $-\mathrm{P}$ & $-\mathrm{T}$ & $-\mathrm{P}$ & & & & - & $\mathrm{P}$ & & & & - & & & & & & & & & \\
\hline Primula malacoides . . . . . . . . . . . . . . . . . . . . & + & - & $+\mathrm{P}$ & $+\mathrm{P}$ & & & $\ldots$ & + & $\overline{-\mathrm{P}}$ & & - & & + & - & & & & & & & & \\
\hline Primula acaulis. . . . . . . . . . . . . . . . . . & $+\mathrm{P}$ & + & $+\mathrm{P}$ & $+\mathrm{P}$ & & & - & - & $\overline{-\mathrm{P}}$ & & - & & - & - & & & & & & & & \\
\hline Cyclamen persicum („Silberlachs“) . . . . . . . . & $+\mathrm{P}$ & + & + & + & & & & & + & & + & & $-\mathrm{P}$ & + & & & & & & & & \\
\hline Syringa vulgaris . . . . . . . . . . . . . . . . . . . & + & + & + & + & & & + & + & & & + & & - & + & & & & & & & & \\
\hline 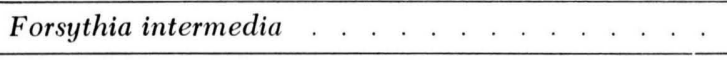 & + & & + & & & & + & + & & + & - & & - & + & & & & & & & & \\
\hline Solanum tuberosum („Oberarmbacher Frühe“) . . . & $-\mathrm{T}$ & $\cdots$ & $\mathrm{T}$ & $-\mathrm{P}$ & & & - & $=$ & - & $\ldots$ & + & - & - & + & - & & & & & & & \\
\hline Solanum lycopersicum („Haubners Vollendung“) . . & - & $-\mathrm{T}$ & $-\mathrm{T}$ & - & & & $-\mathrm{T}$ & & - & $\cdots$ & + & & $\ldots$ & + & 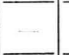 & & & & & & & \\
\hline Calceolaria hybrida . . . . . . . . . . . . . . . . . . . . . . & - & & - & - & & & & & & $\cdots$ & - & & $-\mathrm{T}$ & - & & & & & & & & \\
\hline Columnea hirta. . . . . . . . . . . . . . . . . . . . . & - & $\ldots$ & - & - & & & & - & & $-\mathrm{T}$ & & & $\mathrm{T}$ & $\mathrm{T}$ & - & & & & & & & \\
\hline Cucumis sativus („Hadinger“) . . . . . . . . . . & - & & - & - & & & & $\ldots$ & & & $一$ & & $\cdots$ & & & & & & & & & \\
\hline Cineraria spec. (Randblüte) . . . . . . . . . . . . . . . & & & - & $+\mathrm{P}$ & & & +1 & & & & -1 & & - & $\cdots$ & & & & & & & -1 & \\
\hline
\end{tabular}

Tab. 3. Verteilung gefältelter und nicht gefältelter Cuticula auf den Blütenteilen einiger Dicotylen. 
völlig substanzfrei werden. In solchen Fällen tritt Doppelwandigkeit der Falte primär in der Projektion in Erscheinung (Abb. $2 \mathrm{a}$ ).

Häufig sahen wir, abgesehen von der Fältelung, an den Präparaten eine verhältnismäßig grobe Granulierung. Diese Erscheinung betraf jedoch nicht die morphologische Ausprägung der Cuticula, sondern erfolgte durch Substanzreste, die bei der Präparation nicht restlos entfernt worden waren. An frei von Fremdsubstanzen präparierten Cuticulae war keine Granulierung wahrzunehmen. Der Fortfall der Granulierung galt uns daher als wesentliches Anzeichen der Vollständigkeit des Präparationsvorganges in Kupferoxydammoniak. Die vollständig frei präparierte Cuticula war, von der Fältelung abgesehen, elektronenmikroskopisch in sich stets homogen ${ }^{*}$.

Die sehr unterschiedliche Strukturierung der Cuticulae konnten wir an Hand von etwa 700 elektronenmikroskopischen Aufnahmen und zahlreichen weiteren elektronenmikroskopischen Beobachtungen in Gruppen zusammenfassen.

Bei der Analyse der morphologischen Ausprägung berücksichtigten wir:

I. Die Art der Fältelung.

II. Die Ausbildung der Zellgrenzen.

III. Die Wölbung bzw. Ausstülpung der Cuticula.

Auf Grund dieser Gesichtspunkte konnten wir nachstehende Reihe morphologischer Typen herausarbeiten.

1. Cuticula im gesamten Bereich des Zellumens gefältelt, Zellgrenzen nicht gefältelt (Abb. 3).

2. Cuticula im Bereich des Zellumens gefältelt, Zellgrenzen heben sich durch anders gerichtete Fältelung ab (Abb. 4).

3. Bevorzugte Längs-Richtung der Fältelung der Cuticula, Zellgrenzen abweichend gefältelt oder nicht gefältelt (Abb. 5).

4. Konzentration der Fältelung vorzugsweise im zentralen Bereich des Zellumens, Zellgrenzen deuten nicht auf Wölbung der Zelloberfläche (Abb.6).

5. Cuticula mehr oder minder gewölbter Zelloberflächen im gesamten Bereich des Zellumens gefältelt (Abb. 7).

6. Cuticula mehr oder minder gewölbter Zelloberflächen, Fältelungs-Zentren verschiedenartiger Ausprägung im Bereich der Zellumina (Abb. 8).

${ }^{*}$ Wie wir an anderer Stelle näher ausführen werden, ist auch eine nicht gefältelte Cuticula nicht unbedingt als völlig eben anzusehen, sondern kann, wie beschattete Objekte zeigen, mehr oder minder gewellt sein.
7. Cuticula mit mehr oder minder gefältelten Ausstülpungen im Bereich der Zellumina (Abb. 9).

8. Cuticula durchgehend z. T. unregelmäßig gefältelt, wahrscheinlich ohne Übereinstimmung mit den Zellumina und den Zellgrenzen (Abb. 10).

Die angewandte Präparations- bzw. Untersuchungsmethode beeinflußt die Fältelungsstruktur der Cuticula nicht. Denn das lichtmikroskopische Bild des Abdruckes einer Cuticula turgeszenter Epidermiszellen und das elektronenmikroskopische Bild einer freigelegten Cuticula gleichen Typs lassen erkennen, daß durch die Behandlung mit Kupferoxydammoniak keine Veränderung des Typs der Fältelungsstruktur eintritt. Neuentstehende Falten, die durch Einsinken vorgewölbter turgeszenter Epidermiszellen entstehen, sind als solche, wie bereits erwähnt, bei entsprechender Erfahrung von den nativen Falten zu unterscheiden.

\section{Morphologie der Cuticula einzelner Teile einer Blüte}

Cyclamen persicum. Die Cuticula der Kelchblätter besitzen auf der Ober- und Unterseite eine unterschiedliche Fältelung. Demgegenüber zeigen die Ober- und Unterseite der Blütenblätter ein gleichartig ausgeprägtes Fältelungsmuster. Auf der Blütenblatt-Oberseite hat das Saftmal ein anderes Fältelungsmuster als die Umgebung. Während das Ovarium, abgesehen von den Papillen, eine ungefältelte Cuticula auszeichnet, besitzt der Griffel eine dichte Längsfältelung. Ein auffällig anderes Fältelungsmuster weisen die Staubgefäße auf.

Begonia spec. (weibliche Blüte). Während das Ovarium von einer ungefältelten Cuticula überzogen wird, zeichnen sich Griffel und Narbe sowie Blütenblattober- und Unterseite durch ein besonders zart ausgeprägtes Muster der Fältelung aus.

Rubus fruticosus. Die Cuticula der Kelchblätter ist nicht gefältelt. Die Cuticula der Blütenblatt-Oberseite hat Papillen. Die Blütenblatt-Unterseite ist nicht papillös ausgebildet, sondern besitzt eine Fältelungsstruktur. Das Ovarium hat eine ungefältelte, der Griffel hingegen eine gefältelte Cuticula. Im einzelnen unterscheidet sich bei Griffel und Filamenten das Muster der Fältelung. Auch die Antheren besitzen eine gefältelte Cuticula. Alle Blütenteile der Brombeere zeichnen sich durch Zartheit des Cuticulamusters aus.

Brassica napus. Während sowohl die Innen- als auch die Außenseite der Kronblattnägel von einer 
ungefältelten Cuticula überzogen sind, weisen die Spreiten Fältelung der Cuticula auf. Die Cuticula der Blütenblatt-Oberseite bildet stumpfe Papillen. Epidermiszellen ohne Papillen sind linsenförmig vorgewölbt und zeigen dieselbe Fältelungsstruktur wie die Epidermiszellen der Kronblatt-Unterseite. Das Ovarium besitzt eine ungefältelte, der Griffel hingegen eine gefältelte Cuticula. Die Narbe ist, wie das Ovarium, von einer ungefältelten Cuticula überzogen, doch hat sie eigenartige Trichome, die mit kurzen Borsten versehen sind (Abb. 11). Das Filament besitzt eine Fältelung. Die Antheren weisen gewölbte Epidermiszellen auf; ihr Cuticulamuster kann sehr unterschiedlich sein und von Zelle zu Zelle wechseln. Die Kelchblätter sowie die Nektarien sind von ungefältelten Cuticulae bedeckt.

Azalea spec. Bei dieser Art soll speziell auf die Cuticula-Morphologie der Blütenblätter hingewiesen werden, da hier die inneren Blütenblätter durch Umbildung der Stamina entstanden sind. Die inneren Blütenblätter bilden auf ihrer Ober- und Unterseite dieselben Muster aus wie die äußeren Blütenblätter. Andererseits zeigen die z. T. noch eingewachsenen Antheren eine ungemusterte Cuticula. Es gelang uns Übergangszonen zwischen gefältelten und nichtgefältelten Cuticularegionen an noch nicht vollständig entwickelten inneren Blütenblättern $\mathrm{zu}$ erfassen.

$$
\text { Vergleich der Cuticulae }
$$

Abgesehen von der Tatsache, daß jeder Blütenteil eine fältelungsfreie Cuticula besitzen kann, ist doch die Seltenheit, mit der die Ovarien eine Fältelung der Cuticula aufweisen, sehr auffallend. Auch die Kelchblätter sind nur in wenigen Fällen mit einer gefältelten Cuticula überzogen. Die Blütenblätter sind dahingegen verschiedenartig gemustert. Diese Verschiedenartigkeit bezieht sich z. T. selbst auf Unterschiede des Fältelungsmusters der Ober- und Unterseite des Blütenblattes. Insbesondere sei auf das außerordentliche unterschiedliche Muster auf den verschiedenen Kronblättern der zygomorphen Leguminosenblüte hingewiesen.

Griffel und Filament zeichnen sich in fast allen vor uns beobachteten Fällen dadurch aus, daß die Fältelungsrichtung parallel zur Organachse verläuft.

Narben konnten ihrer Kleinheit wegen nur in wenigen Fällen untersucht werden. Hinzu kam, daß sich bei den Narben die Zellulose nur schwer von der Cuticula löste; mitunter waren die Cuticulahäutchen auch so dünn, daß sie bereits bein Aufbringen auf Objektträger zerrissen.
Bemerkenswert ist die Verschiedenheit der Cuticulamuster auf den Theken. Neben den KronblattCuticulae zeigt dieser Blütenteil die mannigfachste und unterschiedlichste Ausprägung der Fältelung. Auffällig ist dagegen, daß die Konnektive meistens mit einer Cuticula ohne Fältelung überzogen waren.

Übergänge mögen in zwei Fällen näher betrachtet werden. Der Blütenboden der Erdbeere weist neben Zellen, die mannigfaltig gemustert sind, Zellen mit einer Cuticula ohne Fältelung auf. Der Übergang erfolgt nicht plötzlich, sondern zwischen beiden Zonen liegen Zellen mit schwacher Fältelung.

Beim Hafer besitzt die Cuticula der Theken ein besonderes Fältelungsmuster, während das Konnektiv nicht gemustert ist. Wie im ersten angeführten Fall besteht auch hier eine Übergangszone.

\section{Cuticula und Spaltöffnungen}

Auf der Cuticula des Griffels vom Raps fanden wir außer den kleinen Spaltöffnungen, die in gewissen Abständen senkrecht übereinander angeordnet sind, auffallend dünne faltenfreie Stellen. Auch auf der Cuticula des Griffels von Helleborus sahen wir die gleichen Dünnstellen. Aus ihrer Anordnung am Griffel und der Tatsache, daß nach bisherigen Befunden die Cuticula über den Schließzellen sehr dünn beschaffen ist, möchten wir diese Stellen der Cuticula als Zwischenstufen der Entwicklung über angelegten Spaltöffnungen auffassen.

Wir fanden an weiteren Entwicklungsstadien der Cuticula über Spaltöffnungen bereits angedeutete Spalten, die jedoch noch von einem Schließhäutchen verschlossen waren; weiterhin Spalten, über denen das Schließhäutchen bereits aufgerissen war. An vollentwickelten und geöffneten Spalten sahen wir sehr häufig Cutinschläuche verschiedener Länge. Offenbar bilden diese Cutinschläuche das Häutchen, das die Innenseite der Schließ- und Nebenzellen bekleidet. Die Fältelung der Cuticula im Bereich der Spaltöffnungen ist sehr unterschiedlich ausgeprägt.

$$
\text { Cuticulafältelung }
$$

und systematische Verwandtschaft

In seinen Untersuchungen über die Epidermis der Blütenblätter macht $\mathrm{S} \mathrm{chub} \mathrm{e} \mathrm{t^{9 }}$ (S. 238) die Feststellung, daß die Angehörigen einer Gattung eine gewisse Konstanz in der Epidermis-Gestaltung erkennen lassen. Wir kommen hinsichtlich der Cuticulafältelung trotz der verhältnismäßig geringen Zahl

9 K. S c h u bert, Botanisches Arch. 12, 226 [1925]. 
der untersuchten Pflanzen zu dem gleichen Ergebnis. Betrachten wir die nachfolgenden drei Familien, von denen eine größere Anzahl Gattungen untersucht wurde.

I. Ros a cea e. Schubert ${ }^{9}$ (S. 234) teilt diese Familie ein in Gattungen, die auf der Oberseite des Blütenblattes keine echten Papillen tragen (Pirusund Prunus-Arten) und Gattungen, deren Epidermiszellen zu echten Papillen ausgebildet sind ( $u$. a. Rubus und Fragaria-Arten). Dieser Gliederung können wir uns bei der Auswertung der Cuticulafältelung anschließen. Wir fanden bei den bisher untersuchten Prunus-Arten und bei Pirus communis auf der Oberseite der Kronblätter unterschiedliche Fältelungsmuster der Cuticula. Doch besteht eine Ähnlichkeit zwischen den Mustern der Süß- und Sauerkirsche und zwischen denen des Pfirsichs und der Aprikose. Nimmt man aber zum Vergleich der Gattungen nicht nur die Oberseite der Blütenblätter, sondern auch andere Blütenteile, z. B. den Blütenboden hinzu, so ergibt sich eine andere Zusammenstellung der Gattungen innerhalb der Familie, als Schubert sie anführt. Die Blütenboden bei den Prunus- und Fragaria-Arten wird von einer gefältelten, bei den untersuchten Pirus-Arten und bei Rubus fruticosus von einer ungefältelten Cuticula überzogen.

II. Gram in ea e. Den untersuchten Gattungen innerhalb dieser Familie ist gemeinsam, daß weder die Ober- noch Unterseite der Spelzen, noch die Lodiculae und die Ovarien Fältelung der Cuticula aufweisen. Wohl ist aber bei Secale, Triticum, Triticale und Avena die Antheren-Cuticula gefältelt, doch besitzt jede Gattung ihren eigenen Fältelungstyp.

III. L e guminosae. Von den Leguminosen untersuchten wir mehrere Gattungen, aber jeweils nur eine Art. Die Cuticulastruktur der SchiffchenAußenseite sowie -Innenseite ist vergleichsweise bei den einzelnen Gattungen unterschiedlich gestaltet. Die Epidermiszellen von Flügeln und Fahne können, wie $\mathrm{Schubert}^{9}$ (S. 234-235) angibt, Papillen ausbilden. So besitzen bei der Süßlupine die Oberund Unterseite der Fahne und die Flügel-Außenseite, bei der Puffbohne nur die Flügel-Außenseite Papillen.

Wie unterschiedlich die morphologische Ausprägung der Cuticula auf der Innen- bzw. Außenseite der einzelnen Blütenblätter als auch der Fahne, der Flügel und der Schiffchenseiten sein kann, zeigt die Blüte von Wistaria sinensis. Selbst zwischen der violett gefärbten Fahnen-Oberseite und dem auf die- ser sich befindlichen gelb-grünen Saftmal ist ein Unterschied in der Cuticulastruktur zu erkennen.

Zogen wir zum Vergleich der Gattungen innerhalb einer Familie bisher bei den Untersuchungen der Morphologie der Cuticula nur die Blütenblätter, z. T. sogar nur ihre Oberseite, heran, so möchten wir bei dem Vergleich verschiedener Arten einer Gattung weitere Teile der Blüte mit hinzunehmen.

$\mathrm{Rubus}-$ R.idaeus; R. fruticosus. Übereinstimmend ungefältelte Cuticulae haben die beiden Arten über den Ovarien, den Kelchblatt-Innen- und -Außenseiten. Die Cuticula der Blütenbatt-Oberseite der Himbeere sowie der Brombeere ist zu Papillen ausgebildet. Die spezielle Ausbildung der Papillen bei Himbeere und Brombeere ist unterschiedlich. Die Cuticulafältelung der Griffel bevorzugt die Längsrichtung. Grundsätzlich gesehen besteht zwar eine Übereinstimmung des Musters der Cuticulafältelung zwischen Himbeere und Brombeere, jedoch zeigt die Brombeere eine zartere Ausprägung der Strukturen.

B rassica - B.napus; B. rapa. Bei beiden Arten finden wir nach den bisherigen Untersuchungen eine Übereinstimmung in der Cuticulafältelung auf der Oberseite und der Unterseite der Blütenblätter sowie der Antheren. Jedoch ist die Ausprägung der Struktur bei Rübsen erheblich feiner als bei Raps insofern, als das Muster der Fältelung zarter erscheint. Úbereinstimmend haben die Innen- und Außenseite der Kelchblätter, der Blütenblattnägel sowie die Ovarien und die Narben eine ungefältelte Cuticula.

Sol a n u m - S.tuberosum; S. lycopersicum. Bei beiden Solanum-Arten übereinstimmend finden wir - abgesehen von der Ausbildung der Papillen bzw. Trichome - ungefältelte Cuticulae sowohl auf den Kelchblatt- als auch auf den Blütenblatt-Ober- und Unterseiten, auf den Blütenröhren-Außenseiten, den Filamenten, den Ovarien und Narben. Dagegen sind die Griffel und die Theken von einer gefältelten Cuticula überzogen (Tab. 3).

Fragaria $-F$.vesca, diploid; F. vesca, tetraploid; F.orientalis; F. moschata; F.spec. oktoploid. Bei diesen angeführten Fragaria-Arten liegt ebenfalls eine Übereinstimmung in der Ausbildung gefältelter und ungefältelter Cuticulae auf den einzelnen Blütentéilen vor. Auf den Kelchblatt-Unterseiten, den Theken, den Konnektiven, Ovarien und Blütenboden-Außenseiten ist eine ungefältelte Cuticula ausgebildet. Die Cuticula über den Papillen auf der Blütenblatt-Oberseite ist gefältelt; außerdem weisen die Cuticulae auf den Kelchblatt-Oberseiten, den Griffeln und Filamenten in bezug auf die einzel- 
nen Blütenteile unterschiedliche Fältelungsmuster auf.

Aus dieser Gegenüberstellung einzelner Arten einer Gattung kann man doch den Schluß ziehen, daß innerhalb einer Gattung die morphologische Ausprägung der vergleichbaren Blütenteile sehr ähnlich ist. Weitere Analysen in systematischer Richtung wären auf der Grundlage umfangreicher Untersuchungen möglich.

Cuticula der Wildform und Cuticula der Kulturform innerhalb einer Art

Bei Cyclamen persicum liegt zum Vergleich die Cuticula der Kronblatt-Oberseiten von der Kulturform mit großer Blüte und von der Wildform mit kleiner Blüte vor. Beide Formen weisen eine gleichartige Fältelung der Cuticula auf. Aus dieser Tatsache kann man schließen, daß die Ausbildung der Fältelungsmuster erblich fixiert ist. Der Gesamteindruck der Fältelung der Cuticula der Wildform ist nur bedeutend zarter als bei der Kulturform. Die Vermutung liegt nahe, daß eine Korrelation zwischen Organgröße und Feinheit der Cuticulafältelung besteht.

Da wir annahmen, daß die Cuticulafältelung sich u. U. mit der Polyploidie-Stufe ändern könnte, verglichen wir diploide und künstlich erzeugte tetraploide Sippen von Fragaria vesca miteinander. Die Organe, die in der diploiden Stufe eine glatte Cuticula besitzen, haben eine solche auch in der tetraploiden (Ovarien, Theken, Kelchblatt-Unterseiten). Die Cuticula der Kronblatt-Oberseite und der Filamente wurde zum weiteren Vergleich herangezogen. Es ergab sich auch hier, daß zwischen der Ausprägung der Cuticula von di- und tetraploiden FragariaSippen keine Unterschiede beobachtet werden konnten.

Der Deutschen Forschungsgemeinschaft danken wir für die gewährte Sachbeihilfe.

\section{NOTIZEN}

\section{Über Lithium-tetraschwefeltetranitrido-aluminat, $\operatorname{Li}\left[\mathbf{A l}\left(\mathbf{S}_{\mathbf{4}} \mathbf{N}_{\mathbf{4}}\right)\right]$}

Von Margot Goehring und Günter Zirker

Chemisches Institut der Universität Heidelberg

(Z. Naturforschg. 10 b, 58 [1955]; eingeg. am 24. Nov. 1954)

In dem Bestreben, Metallderivate des Tetraschwefeltetraimids, $\mathrm{S}_{4}(\mathrm{NH})_{4}(\mathrm{I})$ herzustellen, setzten wir in Tetrahydrofuran als Lösungsmittel $\mathrm{S}_{4}(\mathrm{NH})_{4}$ mit überschüssigem $\mathrm{Li}\left[\mathrm{AlH}_{4}\right]$ um. Sauerstoff und Feuchtigkeit mußten hierbei sorgfältig ausgeschlossen werden. Es entstanden schon in der Kälte pro $\mathrm{Mol} \mathrm{S}_{4}(\mathrm{NH})_{4}$ etwa $4 \mathrm{Mol} \mathrm{H}_{2}$, und es fiel ein weißer Niederschlag aus, der unter Luftausschluß filtriert und mit Tetrahydrofuran gewaschen wurde. Die Substanz ließ sich im Hochvakuum trocknen und unter reinem Stickstoff unzersetzt aufbewahren; aber schon geringfügige Erschütterungen genügten, um einen von heftiger Detonation begleiteten explosionsartigen Zerfall der Verbindung zu bewirken. Die Analyse der Verbindung ergab die Zusammensetzung $\mathrm{Li}\left[\mathrm{Al}\left(\mathrm{S}_{4} \mathrm{~N}_{4}\right)\right]$. Mit in Ameisen_äure gelöstem KJ setzte sich die Substanz unter Abscheidung von Jod um; nach dem Verdünnen nit Wasser zeigte sich, daß pro $\mathrm{Mol} \mathrm{Li}\left[\mathrm{Al}\left(\mathrm{S}_{4} \mathrm{~N}_{4}\right)\right] 8$ Äquivalente Jod entstanden, und das entspricht einer Oxydationsstufe +2 für den Schwefel in der Verbindung ${ }^{1}$. Bei der vorsichtigen Hydrolyse mit feuchtem Aceton entstand neben $\mathrm{LiOH}$ und $\mathrm{Al}(\mathrm{OH})_{3} \mathrm{~S}_{4}(\mathrm{NH})_{4} .57 \%$ des eingesetzten $\mathrm{S}_{4}(\mathrm{NH})_{4}$ konnten bei der Hydrolyse zurückgewonnen werden. Diese Reaktion zeigt, daß der Tetraschwefeltetraimid-Ring auch in der Komplexverbindung erhalten

1 Zur Bestimmung des Oxydationswertes vgl. M. Go eh ring, Chem. Ber. 80, 110 [1947]. geblieben ist und daß nicht etwa eine Thionitrosyl-Verbindung mit isolierten NS-Gruppen als Ligand vorliegt, wie wir sie von Silber, Kupfer und Thallium kennen?. Der Aluminiumkomplex-Verbindung kommt danach Formel II zu.<smiles>N1SNSNS1</smiles>

I

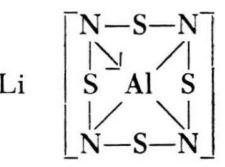

II
Die Analyse der Substanz ergab 2,9\% Li; 12,2\% Al; $25,1 \%$ N. Auf Schwefel wurde nur die noch durch Tetrahydrofuran angefeuchtete Substanz quantitativ untersucht; wir fanden das Verhältnis $\mathrm{Li}: \mathrm{Al}: \mathrm{N}: \mathrm{S}=1: 1: 4,2: 4$. Für $\mathrm{Li}\left[\mathrm{Al}\left(\mathrm{S}_{4} \mathrm{~N}_{4}\right)\right]$ berechnet sich: $3,2 \% \mathrm{Li} ; 12,4 \% \mathrm{Al}$; $25,7 \% \mathrm{~N}$ und $58,8 \% \mathrm{~S}$.

\section{Zur Kristallstruktur und Molekülgestalt des Di-cyclopentadienylmangans}

Von E. We is s und E. O. F is cher

Anorganisch-chemisches Laboratorium der Technischen Hochschule München

(Z. Naturforschg. 10 b, 58-59 [1955]; eingeg. am 4. Jan. 1955)

In Weiterführung röntgenographischer Arbeiten über Metall-dicyclopentadienyle wurde die braune, bei Zimmertemperatur vorliegende Modifikation des $\mathrm{Mn}\left(\mathrm{C}_{5} \mathrm{H}_{5}\right)_{2}{ }^{1}$ untersucht.

1a G. Wilkinson u. F. A. Cotton, Chem. and Ind. 307 [1954].

1b E. O. F i s c h e r u. R. J ir a, Z. Naturforschg. 9 b, 618 [1954]. 